\title{
Development of Hybrid Models for a Vapor-Phase Fungi Bioreactor
}

\author{
Giorgia Spigno $^{1}$ and Stefania Tronci ${ }^{2}$ \\ ${ }^{1}$ Istituto di Enologia e Ingegneria Agro-Alimentare, Università Cattolica del Sacro Cuore, \\ Via Emilia Parmense 84, 29122 Piacenza, Italy \\ ${ }^{2}$ Dipartimento di Ingegneria Meccanica, Chimica e dei Materiali, Università degli Studi di Cagliari, \\ Piazza D’Armi, 09123 Cagliari, Italy \\ Correspondence should be addressed to Stefania Tronci; stefania.tronci@dimcm.unica.it
}

Received 7 December 2014; Accepted 18 May 2015

Academic Editor: Juan A. Almendral

Copyright (C) 2015 G. Spigno and S. Tronci. This is an open access article distributed under the Creative Commons Attribution License, which permits unrestricted use, distribution, and reproduction in any medium, provided the original work is properly cited.

\begin{abstract}
This study is aimed at the development of a model for an experimental vapour-phase fungi bioreactor, which could be derived in a simple way using the available measurements of a pilot-plant reactor, without the development of ad hoc experiments for the evaluation of fungi kinetics and the estimation of parameters related to biofilm characteristics. The proposed approach is based on hybrid models, obtained by the connection of the mass balance equation (used in traditional phenomenological models) with a feedforward neural network (used in black-box modelling), and the proper use of statistical tools for the model assessment and system understanding. Two different hybrid models were developed and compared by proper performance indexes, and their capability to predict the biological complex phenomena was demonstrated and compared to that of a first-principle model.
\end{abstract}

\section{Introduction}

The harmful effects of the emissions of volatile organic compounds (VOCs) on the environment and human health have prompted the development of a wide range of offgas treatment technologies [1]. The degradation of pollutant compounds by means of biological systems is attractive for several reasons: low cost of the process, absence of toxic by-products, ambient condition for operation, and high efficiency of the process.

Among the different biodegradation methods, biofiltration is emerging because of its efficiency to treat large volume of air contaminated by volatile organic compounds (VOCs) [1-3]. In this context, fungi-based biofiltration can support an enhanced mass transfer of hydrophobic VOCs due to the high hydrophobicity of the fungal cell wall and the ability of fungi to colonize with their aerial hyphae the empty space in the biofilter [4]. The possibility of using such processes for industrial applications depends on the availability of good knowledge of the complex phenomena occurring in the biological systems; therefore a considerable effort in bioprocess modeling has been done [5-9]. The implementation of system models is a support for design, management, and process control purposes.

Traditionally, biofilter models are based on mass balance principles and require a good knowledge of the underlying physics of the process such as information on specific growth rate of microbes, biofilm thickness and density, values of diffusivity, partition coefficient, yield, and biofilm distribution [9]. Significant efforts have been done on developing methods and equations to estimate key design parameters of biofiltration processes such as Henry constants, interfacial areas, and active biomass in biofilters [10]. It is worth noticing that the achievement of a precise and reliable model requires a high experimental effort, sometimes with elaborate technical facilities and expertise in order to properly estimate model parameters. For instance, Dorado et al. [11] evidenced that the determination of kinetic parameters is a demanding task, due to the difficulty to reproduce the experimental system and the necessity of calibrating each model for each specific experimental condition. Even if the use of first-principle models proposed in literature is generally able to give a good 
description of the considered biofilter system [8-11], a limited understanding of the biological processes along with the high uncertainties in the model parameter estimation may restrain the applications of such models.

The aim of the present work is to propose a simple modeling approach minimizing the measurement and experimental effort, while retaining model efficiency. Within this purpose, data-driven (or black-box) models are a possible alternative to describe the biological complex systems, with the advantage that, in principle, they can be constructed without any knowledge on the process, if a proper amount of data is available. In particular, neural networks (NNs) have shown good reconstruction capabilities in different application fields [12-15] and, recently, in the modeling of biological wastegas treatment systems [16-21]. As well underlined by Rene et al. [19], the main issues with black-box models concern (i) the availability of a large amount of data from which it is possible to extract the information on the underlying system, (ii) the choice of the network architecture, and (iii) the selection of input variables from which it is possible to infer the process outputs. Sometimes, it may be convenient to take advantage of the a priori knowledge of the nonlinear system using the macroscopic balances (i.e., mass, energy balances) and introducing NNs only for the description of some phenomena. The resulting model is called hybrid $[19,22-24]$, and it has proved to be successful for dynamic systems, with better generalization features and, in addition, identifiable with a reduced set of data with respect to a blackbox equivalent model. To our best knowledge, such hybrid models have been recently applied to waste-water treatment bioreactors [25] and food technology [26, 27] but never to a gas-phase bioreactor.

In previous works $[9,28]$ the authors applied a phenomenological model to a bioreactor, where hexane was removed from a contaminated stream, and the obtained results were compared with experimental data. The biofiltration process was conducted in a vapor-phase fixed-bed bioreactor, containing a biological phase, the fungus Aspergillum niger, immobilized on a support. Different experiments were conducted both on the lab-scale reactor and in batch conditions for assessing, respectively, fluid dynamic properties and kinetic parameters. Even if the first-principle model allowed a good reconstruction of the average removal efficiency of the biofilter at different pollutant loads, model uncertainty was evidenced through sensitivity analysis, showing that partition coefficient, maintenance coefficient, and available specific surface, which had been determined partly by theoretic and simulation approach, were the parameters with greatest influence on the final removal efficiency of the bioreactor.

Following these premises, the aim of this work is to propose an alternative modeling approach based on the available measurements easily acquired in a vapor-phase fungi pilot plant. For this purpose, the first-principle model previously developed [28] has been modified by introducing a neural network for the description of the removal rate of the pollutant, which represents the most difficult modeling task, because it requires the determination of the specific kinetic rate expression (which is usually a complex function of state variables) and of the distribution of the nonuniform biofilm coverage. This information on the reacting biosystem was extracted from the input-output experimental data of the pilot plant, using a neural network in two different hybrid models: (i) a heterogeneous model where the neural network describes the kinetic rate in the biological phase and (ii) a homogeneous model where the neural networks approximate the flux of hexane at the biolayer/air interface. The two different structures have been compared and the assessment of the models has been aided by using proper statistical tools.

\section{Experimental Apparatus and Conditions}

A lab-scale bioreactor inoculated with a strain of Aspergillus niger was used for the treatment of an artificially polluted hexane airstream. The system consisted of two identical columns connected in series; therefore reactor performance could refer either to each single column (single configuration) or to a unique reactor of double length (double configuration). Each column was a jacketed glass column of overall height $0.40 \mathrm{~m}$, internal diameter $25 \mathrm{~mm}$, with a stainless steel net at $40 \mathrm{~mm}$ from the bottom to sustain the packing material (expanded clay in granular form with average $\varnothing 3-5 \mathrm{~mm}$ ) and sampling ports for the substrate and air supply and for the outlet gas flow. The contaminated air-stream was artificially created by mixing two distinct flows supplied by a compressor: the first one was passed through a humidifying system; the second one was made air sparging in a vessel containing liquid hexane at $30^{\circ} \mathrm{C}$. The gas flow rate was set to $4 \cdot 10^{-3} \mathrm{~m}^{3} / \mathrm{h}$, corresponding to an empty bed residence time (EBRT) of $159 \mathrm{sec}$. Optimal EBRT should be in the range from 15 to $60 \mathrm{sec}$, but the minimum value is actually dictated by the given set of off-gas composition and filter conditions, the pollutant RE, or maximum outlet concentration allowed by regulations. In our case preliminary trials with a double flow rate and corresponding EBRT of $80 \mathrm{sec}$ had brought to an almost zero RE and poor mycelium development [28].

Even if the system was located in a conditioned room to work as close as possible to a constant temperature of $30^{\circ} \mathrm{C}$, optimal value for the fungal growth, daily monitoring temperature showed that it varied from $19^{\circ} \mathrm{C}$ to $30^{\circ} \mathrm{C}$.

More details about the bioreactor inoculation procedure, the development of the experiments, and the results obtained from this reactor were already reported $[9,28]$.

Reactor performance was represented in terms of removal efficiency (RE) calculated from the inlet $\left(C_{G 0}\right)$ and outlet $\left(C_{G e}\right)$ gas concentration, as reported in the following:

$$
\mathrm{RE}=\frac{C_{G 0}-C_{G e}}{C_{G 0}} \text {. }
$$

\section{Modeling Approach}

Starting from the first-principle model of the biofiltration system, a hybrid model approach has been proposed where empirical models (e.g., neural networks) are used to describe the most critical phenomena of the considered biosystem.

3.1. First-Principle Model. Before describing the hybrid models developed in the present paper, the first-principle model 
previously proposed [9] to describe the biofiltering system of Section 2 is recalled:

$$
\begin{aligned}
& \frac{D v}{H^{2}} \frac{\partial^{2} S_{G}}{\partial \zeta^{2}}-\frac{U_{g}}{H} \frac{\partial S_{G}}{\partial \zeta}+\left.\frac{D_{e} \alpha A}{\delta^{*}} \frac{\partial S_{F}}{\partial \eta}\right|_{\eta=0}=0 \\
& \frac{C_{G 0} D_{e}}{\delta^{* 2}} \frac{\partial^{2} S_{F}}{\partial \eta^{2}}-\frac{X_{F} \mu_{\max }}{Y} \frac{S_{F}}{\left(K_{S} / C_{G 0}+S_{F}+C_{G 0} S_{F}^{2} / K_{I}\right)} \\
& \quad-X_{F} m_{s}=0
\end{aligned}
$$

with the following boundary conditions:

$$
\begin{aligned}
& \zeta=0,0 \leq \eta \leq 1, \quad \frac{D v}{U_{g} H} \frac{\partial S_{G}}{\partial \zeta}=S_{G}-1 \\
& \zeta=1,0 \leq \eta \leq 1, \quad \frac{\partial S_{G}}{\partial \zeta}=0 \\
& \eta=0,0 \leq \zeta \leq 1, \quad S_{F}=\frac{S_{G}}{m} \\
& \eta=1,0 \leq \zeta \leq 1, \quad \frac{\partial S_{F}}{\partial \eta}=0 .
\end{aligned}
$$

In the above equations $S_{G}$ and $S_{F}$ are the dimensionless pollutant concentrations, respectively, in the gas phase and in the biofilm phase, obtained by dividing the actual concentrations, $C_{F}$ and $C_{G}$, for the inlet concentration $C_{G 0} ; U_{g}$ is the superficial gas velocity; $\zeta$ is the dimensionless reactor height calculated with respect to the reactor length $(h / H$, with $h$ being the position in the column); and $\eta$ is the dimensionless biolayer thickness calculated with respect to the effective one $\left(\theta / \delta^{*}\right.$, with $\theta$ being the position in the biolayer and $\delta^{*}$ the effective biolayer thickness). The model has six parameters related to the system fluid dynamics, biofilm characteristics, and mass transfer: $D$ is the dispersion coefficient in the reactor; $v$ is the bed porosity; $D_{e}$ is the effective diffusion coefficient of the pollutant in the biolayer; $A$ is the biolayer surface area per unit volume of the reactor; $\alpha$ is the fraction of $A$ covered by the biofilm; and $m$ is the pollutant air/biofilm distribution coefficient. For the specific growth rate expression of the fungi growing on hexane, Monod kinetics with an Andrews type inhibition was assumed where $K_{S}$ is the saturation constant; $K_{I}$ is the inhibition constant; $m_{S}$ is the maintenance coefficient; $\mu_{\max }$ is the maximum specific growth; $X_{F}$ is the biofilm density; and $Y$ is the biomass yield coefficient.

The model has many parameters, which had been evaluated [9] partly through a trial-and-error method, partly using the knowledge on the process obtained through experimental data and from the literature (Table 1). The proposed model showed good performance capabilities, but sensitivity analysis evidenced model uncertainty, principally due to parameters related to the biological phase, which are difficult to be experimentally evaluated. The main critical experimental points concern the evaluation of biodegradation kinetics and the prediction of biomass film distribution, which strongly influence degradation and imply large data variability. This aspect unfavorably plays in the construction of a bioreactor
TABLE 1: Estimation of parameters in the first-principle model.

\begin{tabular}{lc}
\hline Model parameter & Estimation \\
\hline$D$ & Experimental \\
$D_{e}$ & Theoretical \\
$\alpha$ & Experimental/theoretical \\
$V_{\text {biomass }}$ & Experimental/theoretical \\
$\Delta$ & Experimental/theoretical \\
$K_{S}$ & Experimental \\
$X_{f} \cdot \mu_{\max } / Y$ & Experimental \\
$K_{I}$ & Theoretical/simulation \\
$m_{S}$ & Theoretical/simulation \\
$m$ & Simulation \\
\hline
\end{tabular}

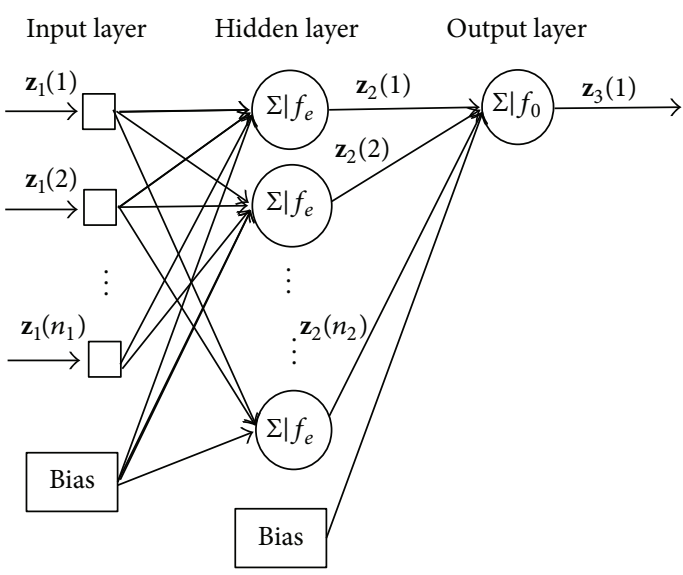

Figure 1: Scheme of the feedforward neural network.

model, which should be robust with respect to data variation or presence of outliers. It is also important to notice that some parameters are quite difficult to be experimentally estimated, like the partition coefficient $m$, while biofilm thickness and biofilm surface are impossible to measure and might be only adjusted by fitting. Furthermore, the inoculated fungus Aspergillus niger can develop as filamentous mycelium and spores, and in the previous study it was not possible to recognize which form was responsible for hexane degradation.

3.2. Neural Networks. A feedforward neural network (FNN) has been used here coupled with the first-principle model, in order to describe phenomena occurring in the biofilter systems and related with pollutant degradation kinetics. A general FNN is represented in Figure 1, for $n_{1}$ inputs, represented by the vector $\mathbf{z}_{1}$, and one output, $\mathbf{z}_{3}$. In more detail, the input signals $\mathbf{z}_{1}$ are scaled by the adjustable parameters, called weights, $w_{1}(i, j)$; then all the contributions are summed and processed by the activation function $f_{e}(3 \mathrm{a})$. The resulting signal, $\mathbf{z}_{2}$, is scaled by the weight $w_{2}(i, j)$. Its components 
are summed and mapped into the vector $\mathbf{z}_{3}$ by the activation function $f_{0}(3 \mathrm{~b})$ :

$$
\begin{aligned}
& z_{2}(i)=f_{e}\left(\sum_{j=1}^{n_{1}} w_{1}(j, i) z_{1}(j)+w_{1}\left(n_{1}+1, i\right) b\right) \\
& z_{3}(i)=f_{o}\left(\sum_{j=1}^{n_{2}} w_{2}(j, i) z_{2}(j)+w_{2}\left(n_{2}+1, i\right) b\right),
\end{aligned}
$$

where $b$ represents the bias term.

Neural networks are data-driven models and, in principle, it is not necessary to have a deep knowledge on the physicochemical phenomena governing the process. They are universal approximators [29]. Therefore they should be able to model any nonlinear system if the proper network structure is used, that is, the right network input variables and the number of hidden neurons.

In this work, a knowledge-based approach supported by statistical tools has been used to identify the inputs of the network, which are the variables affecting the consumption of reactant. With regard to hidden neurons, it is important to underline that they cannot be determined from the knowledge of the process, because they elaborate signals that have lost the physical meaning of the inputs. In this case, a trialand-error method has been used for the choice of the number of hidden units: starting from the general consideration that a parsimonious model is preferred, the number of hidden units has been evaluated from the simplest model, with only one hidden neuron and adding one more neuron until a significant change in the model performance was observed. Then, the input and hidden layers were augmented with an extra neuron, the bias, which provides a constant output signal equal to one.

3.3. Hybrid Models. Considering the drawbacks underlined for the first-principle model in Section 3.1, the aim of the present work is finding a simple modeling approach, which could be easily applied also to industrial bioreactors, where the possibility of accomplishing experimental measurements for biomass characterization is scarce. The approach proposed here is based on hybrid models, obtained by the integration of a FNN in the first-principle model ((2a)(2c)). Aiming at the best compromise between simplicity of description and prediction capabilities of the model, two different approaches have been considered to reconstruct the biological system behavior: a heterogeneous and a homogenous model.

3.3.1. Heterogeneous Hybrid Model (GM1). The identification of the kinetic law occurring in the biological phase is one of the most critical points when describing biological systems, because the kinetic constants may vary significantly with process conditions [30]. The neural network, indicated as $f_{\mathrm{NN} 1}\left(\mathbf{z}_{1}\right)$, has been therefore introduced in the reactor model $((2 b)-(2 c))$ to estimate hexane reaction rate along with the term $X_{F} m_{s}$; therefore the following heterogeneous or twophase hybrid model is obtained ((4a) and (4b)):

$$
\begin{aligned}
\frac{D v}{H^{2}} \frac{\partial^{2} S_{G}}{\partial \zeta^{2}}-\frac{U_{g}}{H} \frac{\partial S_{G}}{\partial \zeta}+\left.\frac{D_{e} \alpha A}{\delta^{*}} \frac{\partial S_{F}}{\partial \eta}\right|_{x=0}=0 \\
\frac{C_{G}(0) D_{e}}{\delta^{* 2}} \frac{\partial S_{F}}{\partial \eta^{2}}-f_{\mathrm{NN} 1}\left(\mathbf{z}_{1}\right)=0
\end{aligned}
$$

along with the boundary conditions reported in Section 3.1. In the above formulation $\mathbf{z}_{1}$ is the input vector of the network (cf. Figure 1), which consists of independent selected variables affecting the reaction rate. It will be defined later.

The use of a neural model has the immediate consequence that it can be applied directly on the experimental data obtained in the reactor configuration used for the biofiltration of the polluted stream, without the necessity of conducting ad hoc experiments for kinetics identification and parameter estimation, as those previously carried out [9].

3.3.2. Homogeneous Hybrid Model (GM2). A further simplification of the model has been obtained by using the neural network to describe the flux of the hexane at the gasbiological phase interface, avoiding also the estimation of the parameters related to the biofilm characteristics. As a result, a homogeneous model is obtained, where the derivative of concentration in the biological phase is modeled by a neural network, indicated as $f_{\mathrm{NN} 2}\left(\mathbf{z}_{1}\right)$, as reported in

$$
\frac{D v}{H^{2}} \frac{\partial^{2} S_{G}}{\partial \zeta^{2}}-\frac{U_{g}}{H} \frac{\partial S_{G}}{\partial \zeta}+f_{\mathrm{NN} 2}\left(\mathbf{z}_{1}\right)=0
$$

In this case only the fluid dynamic characteristics of the system need to be estimated, and the reactor efficiency is obtained by integrating (5) along with the boundary condition with respect to $\zeta$, reported in Section 3. Again, the input network variables, indicated with $\mathbf{z}_{1}$, will be defined later.

\section{Development of the Neural Network}

The experimental data available for parameter estimation and neural model validation were 290 outlet concentration values, at constant gas flow rate of $4 \cdot 10^{-3} \mathrm{~m}^{3} \mathrm{~h}^{-1}$, according to the single and double configuration, at different inlet concentration and temperature values, spanning, respectively, from 1 to $20 \mathrm{~g} \mathrm{~m}^{-3}$ and from 19 to $30^{\circ} \mathrm{C}$. All the used concentration values were collected at regime condition, that is, when, after an adaptation period of about two weeks, biomass development was not visually observed anymore and steadystate conditions could be assumed [9].

Data have been randomized and divided into training (90\%) and test (10\%) set. The former series has been further divided into two different sets, one set for parameter estimation (80\%) that means the proper training and one set $(10 \%)$ for cross-validation. The latter data set is used in order to assure generalization capability of the neural model [29]. In more detail, the training phase is stopped when the objective function calculated on the cross-validation data reaches the minimum. 
The number of data points used for the development of the hybrid models is on average a high number compared to the other artificial neural network models developed for different waste-gas treatment systems [19] and it matches the requisite that data-driven model needs a high number of experimental patterns in order to give a good estimate of the process.

The training of the network has been developed by means of the Levenberg-Marquardt optimization algorithm. Unlike more common applications of neural network modeling, the variables to be estimated, which are reaction rate (GM1) and pollutant flux at the air/biolayer interface (GM2), are not experimentally measured; therefore FNN parameter estimation has been based on the error between the experimental and calculated concentration at the reactor exit. The latter is obtained through the integration of the reactor model, assuming that the error between the experimental and calculated concentration is exclusively due to reaction rates for GM1 and due to the hexane flux for GM2. In particular, the following objective function $\Phi(\mathbf{w})$ has been used for both models:

$$
\Phi(\mathbf{w})=\sum_{i=1}^{N_{t}}\left[\frac{\left(C_{G}^{e}(i)-C_{G}^{c}(i)\right)}{C_{G}^{e}(i)}\right]^{2},
$$

where $\mathbf{w}$ represents the weights (parameters) of the neural model, $N_{t}$ is the number of data used for the training, and $C_{G}^{e}(i)$ and $C_{G}^{c}(i)$ are, respectively, the experimental and calculated concentration at reactor exit.

The minimum search was accomplished for every network structure considering one hundred initial $\mathbf{w}$ vector values, randomly generated, and considering those weights leading to the lowest error calculated on cross-validation set. This approach solves the problem of generalization, that is, the obtainment of good performance with data that do not belong to the training set [29]. The cross-validation was also used to examine the prediction capabilities of neural network with respect to sample variation and to assess model robustness.

The development of the neural kinetic model should capture, as well as possible, the essential characteristics of the functional relationships between inputs (i.e., concentration and temperature) and outputs (reaction rate). In other words, the neural kinetic model must also provide consistent derivatives of the reaction rate with respect to the concentration and temperature.

\section{Selection of the Model}

The construction of the two neural models has been accomplished selecting the model inputs, the number of hidden neurons, and the activation functions. The selection of the best structure has been based on the following performance indexes evaluated on the training data: (i) Coefficient of determination $R^{2}$, which measures the variance explained by the model and defined as

$$
R^{2}=1-\frac{\sum_{i=1}^{N_{t}}\left(C_{G}^{e}(i)-C_{G}^{c}(i)\right)^{2}}{\sum_{i=1}^{N_{t}}\left(C_{G}^{e}(i)-\bar{C}_{G}\right)^{2}},
$$

where $\bar{C}_{G}$ is the average concentration value calculated for the $N_{t}$ experimental points of the training set;

(ii) Standardized residuals, $d(i)$, which indicates if there are deterministic features that have not been predicted by the model, defined as [31]

$$
d(i)=\frac{\left(C_{G}^{e}(i)-C_{G}^{c}(i)\right)}{\sqrt{\left(1 /\left(N_{t}-N_{w}\right)\right) \sum_{i=1}^{N_{t}}\left(C_{G}^{e}(i)-C_{G}^{c}\right)^{2}}},
$$

where $N_{w}$ is the number of neural model parameters. Nonrandom behavior of residuals with respect to calculated RE reveals the inadequacy of the model to capture system behavior. The Kolmogorov-Smirnov test is also used to assess the goodness of fit [32-34].

5.1. Selection of NN Structure in GM1. The use of neural network in the two-phase model GM1 allows using a priori information on the reactor fluid dynamics and biofilm phase. In particular, the following parameters previously reported [9] have been used: $D, D_{e}, A, \alpha, \delta^{*}$, and $m$. The kinetic law has been, on the other hand, extracted from the experimental data using the proper input variables, in particular hexane concentration in the biofilm and reactor temperature. The results obtained for the training showed that temperature does not affect the reaction rate in the considered experimental conditions, confirming the correctness of isothermal assumption in the phenomenological model [9].

The selection of the hidden neurons has been established by varying the number of neurons in the range 1-4 and the best structure, consisting of two hidden neurons, has been selected on the basis of residual analysis and $R^{2}$. Training results evidenced better model behavior when a sigmoidal and a linear activation function were used, respectively, for input and hidden neurons instead of using the nonlinear one for both the layers. The analytical forms of the activation functions are reported in

$$
\begin{aligned}
f_{e} & =\frac{1}{1+e^{-\left(\sum_{j=1}^{n_{1}} w_{1}(j, i) \mathbf{z}_{1}(j)+w_{1}\left(n_{1}+1, i\right) b\right)}} \\
f_{o} & =\sum_{j=1}^{n_{2}} w_{2}(j, i) \mathbf{z}_{2}(j)+w_{2}\left(n_{2}+1, i\right) b .
\end{aligned}
$$

A summary on the structures and performance indexes for different network is reported in Table 2, while Figure 2(a) shows the results obtained for the training data set in terms of comparison between experimental removal efficiency (RE) and calculated ones.

It is worth noting that the variability of the data is quite large due to the variations of biomass activity with respect 
TABLE 2: Network structure and performance indexes of the two hybrid models.

\begin{tabular}{|c|c|c|c|c|c|c|c|c|}
\hline \multirow{2}{*}{ Model } & \multirow{2}{*}{ Model inputs } & \multirow{2}{*}{ Needed parameters } & \multirow{2}{*}{ Input neurons } & \multirow{2}{*}{ Hidden neurons } & \multicolumn{2}{|c|}{ Training } & \multicolumn{2}{|c|}{ Test } \\
\hline & & & & & $R^{2}$ & MSE & $R^{2}$ & MSE \\
\hline GM1 & $C_{F}$ & $D, D_{e}, A, \alpha, \delta^{*}, m$ & 1 & 2 & 0.824 & 0.0076 & 0.83 & 0.0059 \\
\hline GM2 & $C_{G 0}, C_{G}$ & $D$ & 2 & 2 & 0.844 & 0.0067 & 0.88 & 0.004 \\
\hline
\end{tabular}

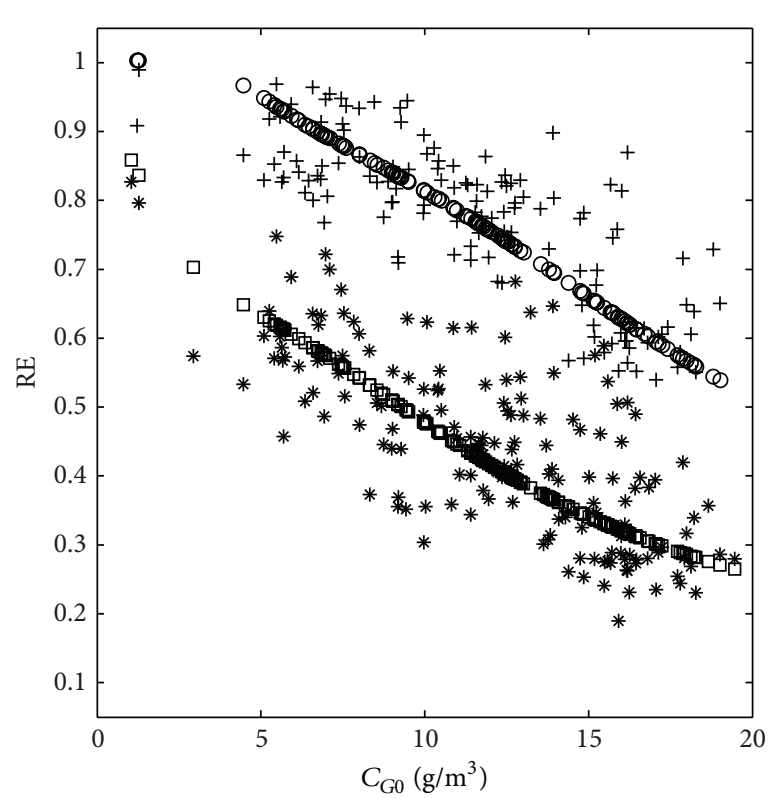

(a)

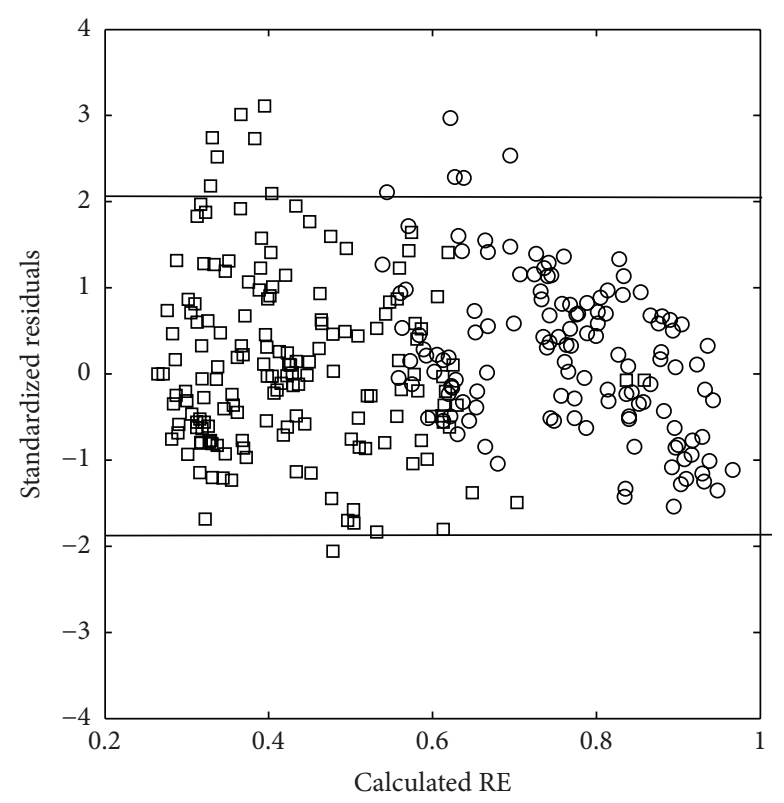

(b)

Figure 2: Training results (removal efficiency RE versus inlet pollutant concentration $C_{G 0}$ ) for heterogeneous hybrid model GM1: (a) comparison between experimental (one reactor configuration: $[*]$, two reactors' configuration: $[+]$ ) and GM1 data (one reactor configuration: [ $\square$ ], two reactors' configuration: [O]) and (b) standardized residuals with respect to predicted RE (one reactor configuration: [ $\square$ ], two reactors' configuration: [O]).

to inlet pollutant concentration, as evidenced in [28]. The hybrid model GM1 follows a medium trend of the RE with respect to the inlet hexane concentration. Modeling errors may exceed the measurement error that is equal to $5 \%$, but it is important to underline that the estimated RE correctly tends to unity, for both reactor configurations, as $C_{G 0}$ tends to zero.

Furthermore, a measure of the quality of the fitting is obtained plotting the standardized residuals with respect to the calculated RE (Figure 2(b)). The trend appears without a deterministic structure, indicating that the obtained model captures the essential features of the data. The data contained in the region $(-2,2)$ are more than the $95 \%$ of the total amount of data used for training, indicating that residuals can be reasonably modeled as an outcome of a random normal value. This is also corroborated by the Kolmogorov-Smirnov test $[33,34]$ which does not reject the null hypothesis of Gaussian assumption of the residuals with a significance level of $5 \%$.

5.2. Selection of the NN Structure for GM2. The neural network used in the homogeneous model has to describe two steps of the hexane degradation; in particular it should extract from the experimental data the phenomenon of both adsorption and reaction in the biological phase. On the other hand, GM2 needs less information on the biological phase and kinetics, therefore requiring a minor experimental effort in terms of ad hoc experiments and sophisticated system analysis. In particular, only the dispersion coefficient $(D)$ has been used (5).

The selected network inputs are inlet hexane concentration and concentration of hexane along the reactor. Again, results do not evidence temperature effects on $\mathrm{RE}$, leading to a network with two inputs.

The number of hidden neurons leading to the best results has been, in this case, equal to two and, again, a sigmoidal and a linear activation function have been used, respectively, in the input and hidden neurons.

The principal aspects of GM2 model are reported in Table 2, and the results for the training set are shown in Figure 3(a). As for GM1, modeling errors may be higher than measurement errors, but it is important to note that residuals (Figure 3(b)) again seem randomly disposed with respect to the estimated variable, and the amount of residuals enclosed in the $(-2,2)$ region is less than $95 \%$. Applying the Kolmogorov-Smirnov test $[33,34]$ to residuals with a significance level of $5 \%$ the null hypothesis of Gaussian assumption is again not rejected. 


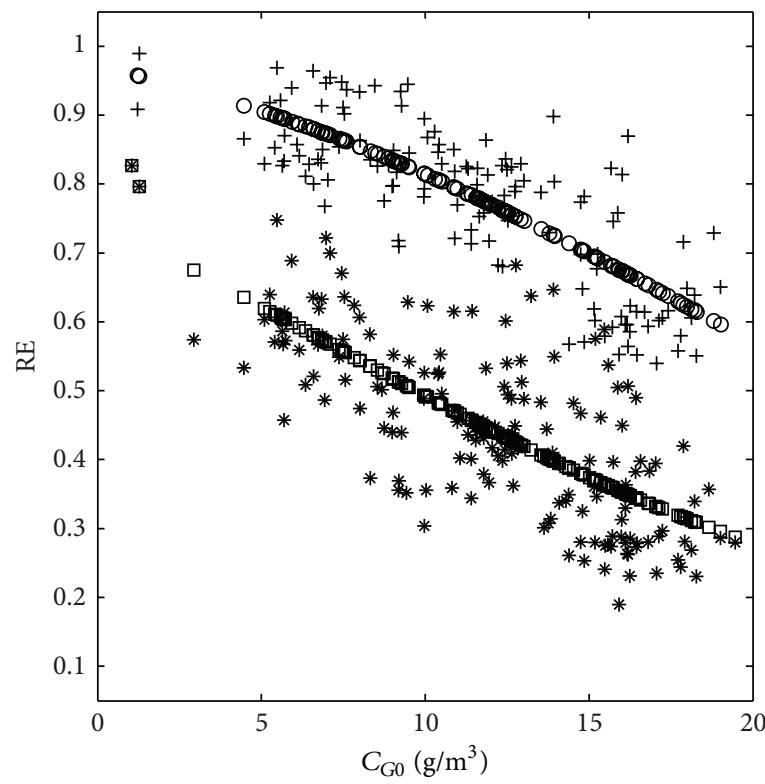

(a)

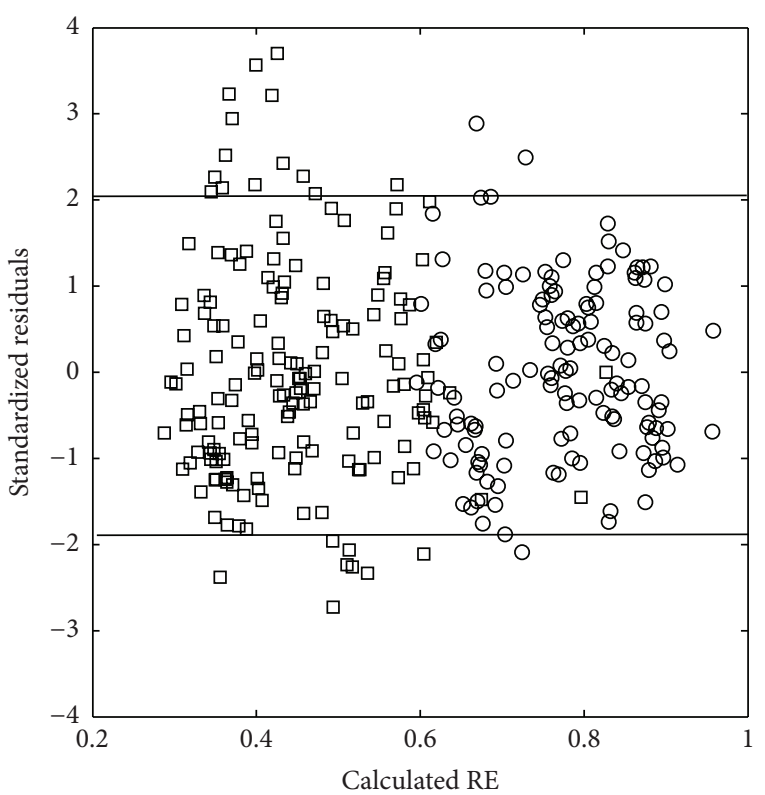

(b)

FIgURE 3: Training results (removal efficiency RE versus inlet pollutant concentration $C_{G 0}$ ) for homogeneous hybrid model GM2: (a) comparison between experimental (one reactor configuration: [*], two reactors' configuration: $[+]$ ) and GM2 data (one reactor configuration: [ $\square$ ], two reactors' configuration: $[O]$ ) and (b) standardized residuals with respect to predicted RE (one reactor configuration: [ $\square$ ], two reactors' configuration: $[\mathrm{O}])$.

\section{Test Results}

The ability of the two hybrid models to predict the biofiltration system behavior has been evaluated by comparing calculated values with the experimental data selected for testing purposes. To analyze the model performance for a wide range of pollutant inlet concentration, the interval of variation of $C_{G 0} 1-20 \mathrm{~g} \mathrm{~m}^{-3}$ has been divided into nineteen subintervals of unitary length. Test data $(10 \%$ of all the data points) have been randomly selected with more than one sampling from intervals with larger amount of data. Because those data have not been used during the network training, this comparison shows the performance of the developed models in unknown situations.

The test of GM1 is reported in Figure 4, where the removal efficiency calculated with the hybrid model for the single (Figure 4(a)) and double (Figure 4(b)) configuration reactor is compared with the experimental data, with a mean square error for the total points equal to 0.0059. Considering the significant variability of the experimental RE used for training and validation of the model, the capability reconstruction of the hybrid model is quite good. This is also evidenced by the closeness of GM1 estimation with the RE calculated using the first-principle model [28], which has a mean square error equal to 0.0064 . This means that the information contained in the data available at reactor exit allows a good reconstruction of the kinetic law, which rules hexane degradation in the biofilm phase. As a further confirmation of the capability of the hybrid model approach, the kinetic rate predicted by the neural model and that obtained in [9] (cf. (2b)) are reported in Figure 5. Even if a mismatch exists in the estimation of the two models, the qualitative behavior of the kinetic law is well reproduced using only concentration data calculated in one point of the reactor. This result indicates the possibility of using this approach to obtain information on the functional form of a kinetic law, when this is unknown for the problem at hand.

The GM2 test results are reported in Figures 6(a) and 6(b), where the comparison with the experimental data shows again discrete performance of the model. In this case the distance between the hybrid model and the experimental data is smaller than in the previous case, the mean square error being equal to 0.004 , but there is bigger mismatch with the firstprinciple model. This is because GM2 used only information on the system fluid dynamics, extracting all the information regarding biofilm characteristics, kinetics, and mass transfer phenomena from the experimental data (Table 2), with the advantage of reducing ad hoc experiments and avoiding the use of difficult analytical procedures to calculate the model parameters related to the biofilm characteristics.

\section{Conclusions}

The modeling problem of a gas-phase bioreactor was solved by resorting to hybrid models, a combination of material balances (used in traditional phenomenological models) and neural networks used to describe the most complex phenomena present in the process. Two different options were investigated: (i) a heterogeneous model where the neural network describes the rate of consumption of the pollutant in the biological phase and (ii) a homogenous model where the neural network reconstructs the flux of the hexane at 


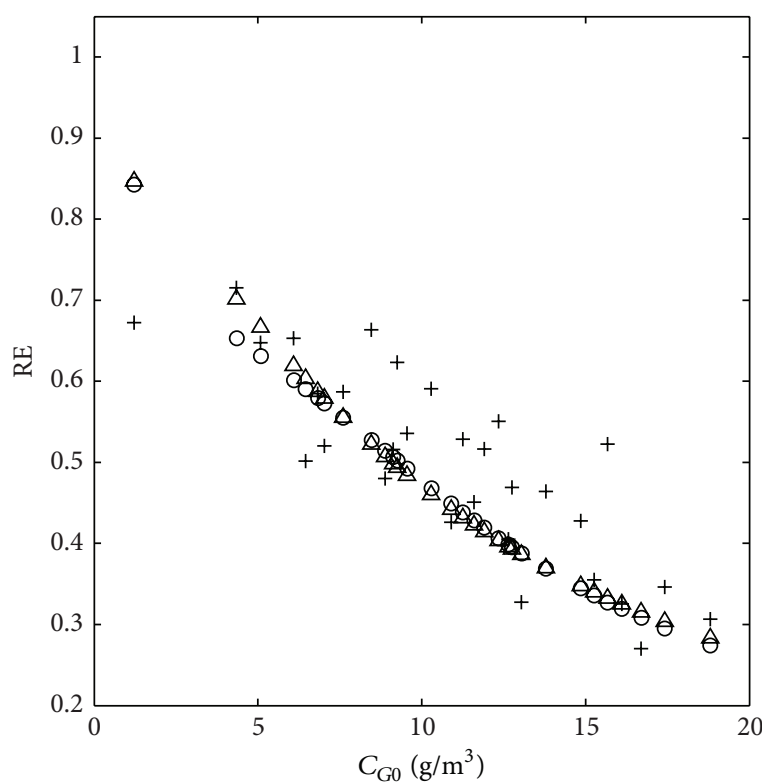

(a)

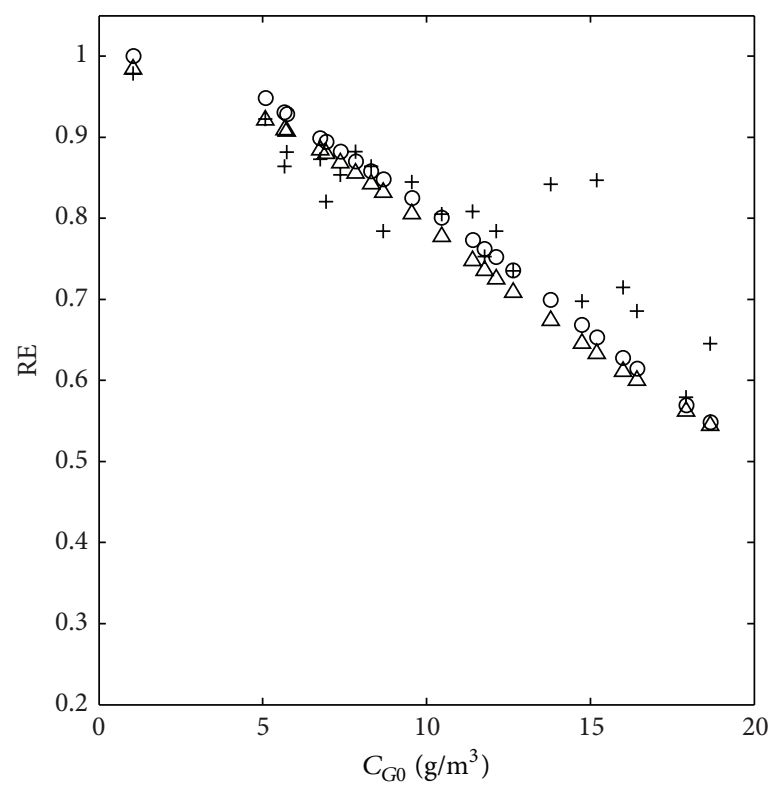

(b)

Figure 4: Test results (removal efficiency RE versus inlet pollutant concentration $C_{G 0}$ ): comparison among heterogeneous hybrid model [O], first-principle model $[\Delta]$, and experimental data $[+]$ for (a) single and (b) double configuration reactor.

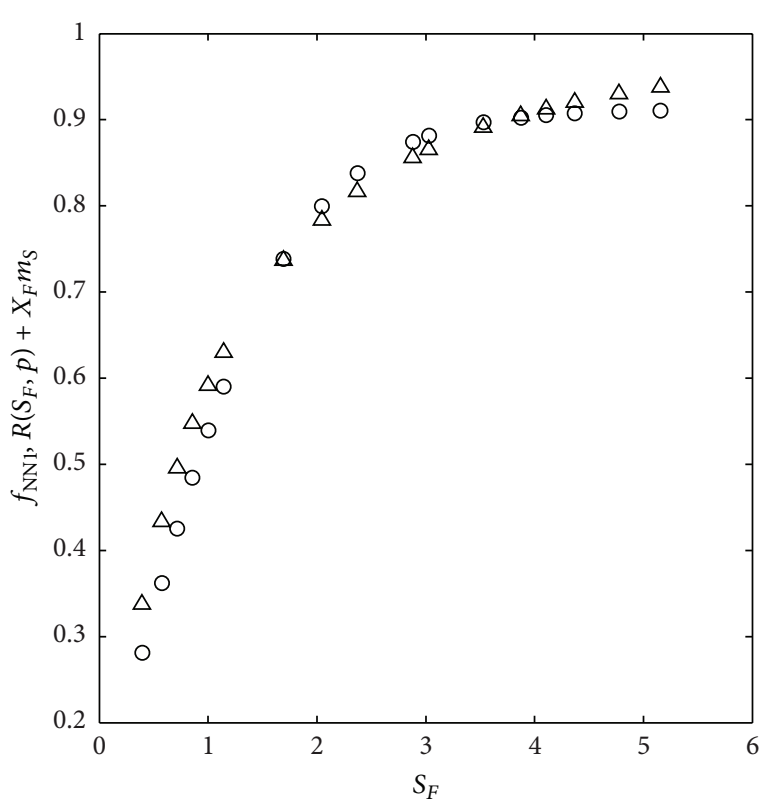

FIGURE 5: Comparison between kinetic rate calculated with the phenomenological model as $\left(X_{F} \mu_{\max } / Y\right)\left(S_{F} /\left(K_{S} / C_{G 0}+S_{F}+\right.\right.$ $\left.\left.C_{\mathrm{G} 0} S_{F}^{2} / K_{I}\right)\right)[\Delta]$ and neural network $\left(f_{\mathrm{NN} 1}\right)$ plus the constant term $X_{F} m_{s}[\mathrm{O}]$ as a function of dimensionless pollutant concentration in the biological phase $\left(S_{F}\right)$.

the interphase, avoiding the integration of the mass balance in the biological phase. Model construction was based on a large amount of experimental data, characterized by a large variability, from which both the hybrid models were able to capture the deterministic features of the observations (as demonstrated by residual analysis). It is worth noting that the neural model has not been used in the traditional way, where it is trained to model directly observed variables. In fact, in the present paper the kinetic rate or the mass transfer term was not measured; therefore the neural networks had to learn their functionality by using an indirect measure, which was the outlet pollutant concentration.

The main contribution of the proposed modeling approaches is the reduction of time and resources necessary to conduct experimental activities aimed at the identification of kinetic law and mass transfer phenomenon for the bioreactor, which are the most demanding task when modeling biological systems. The satisfactory reconstruction capabilities of the neural networks in both the heterogeneous and homogeneous model have been demonstrated by comparing hybrid models predictions with the ones obtained using the first-principle model. The good match between the predictions indicates that neural networks are able to extract information on specific complex phenomena from the simple observations of the laboratory reactor exit concentration. Furthermore, through the analysis of residuals [31, 32] and Kolmogorov-Smirnov [33, 34] test applied to the hybrid models, it was possible to corroborate the isothermal assumption used in the phenomenological model.

Results evidenced that the behavior of the heterogeneous model was closer to that of first-principle model compared to the homogenous one, with good prediction of the reaction rate law. This model should, then, be recommended. On the other hand, the homogenous model used less a priori information on the biosystem, which might imply a possible reduction of performance when used at different operating conditions (e.g., changes of gas flow rate). To overcome this 


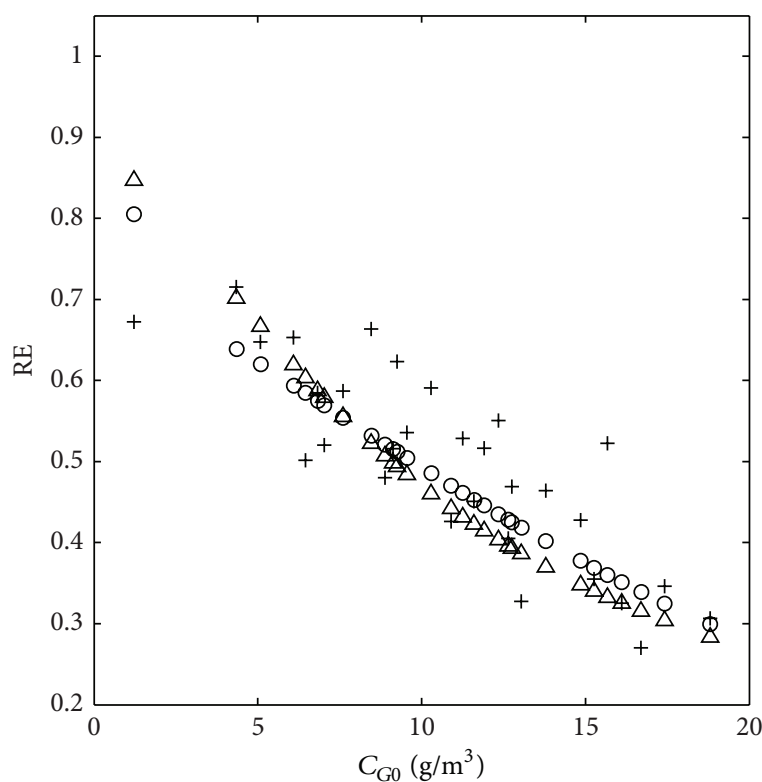

(a)

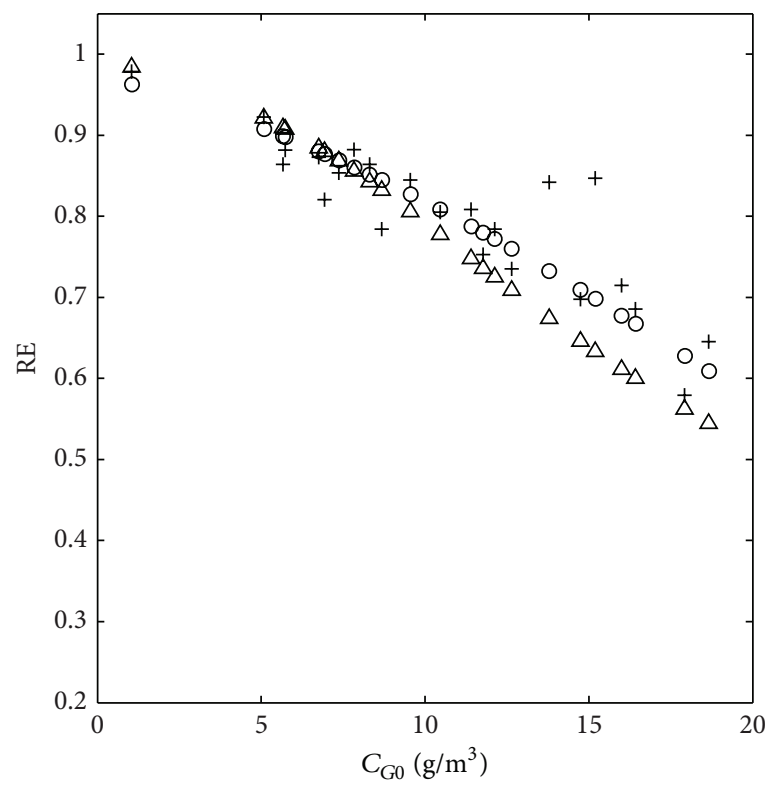

(b)

Figure 6: Test results (removal efficiency RE versus inlet pollutant concentration $C_{G 0}$ ): comparison among homogeneous hybrid model [O], first-principle model $[\Delta]$, and experimental data $[+]$ for (a) single and (b) double configuration reactor.

limitation, a proper experimental campaign (i.e., with ad hoc trials at different flow rates) followed by model recalibration could be carried out to improve description model capabilities and obtain a wider applicability. This approach is easier to be implemented in a full-scale industrial plant making the homogenous model preferable to the heterogeneous one.

The proposed methodology allows the construction of parsimonious models, which are useful for on-line application, and also requires less computational time during training. In particular, hybrid models based on neural networks can be useful for process monitoring purposes in case of delayed concentration measurements, where it is advantageous to have a simple tool to fast recognize if the system is going out of control and take corrective measures. Furthermore, the availability of the model can give an effective tool for on-line optimization of the process.

\section{Nomenclature}

A: Biolayer surface area per unit volume of the reactor $\left(\mathrm{m}^{-1}\right)$

$C_{G}$ : Concentration of the pollutant in the air at position $h$ along the biofilter $\left(\mathrm{g} / \mathrm{m}^{3}\right)$

$\bar{C}_{G}$ : Average concentration value calculated for the experimental points of the training set $\left(\mathrm{g} / \mathrm{m}^{3}\right)$

$C_{G}^{e}$ : Experimental concentration

$C_{G}^{c}$ : Calculated concentration

$C_{G 0}$ : Concentration of the pollutant in the air at the inlet of the biofilter $\left(\mathrm{g} / \mathrm{m}^{3}\right)$

$C_{G e}$ : Concentration of the pollutant in the air at the outlet of the biofilter $\left(\mathrm{g} / \mathrm{m}^{3}\right)$

$C_{F}$ : Concentration of the pollutant at a position $\theta$ in the biolayer at a point $h$ along the column $\left(\mathrm{g} / \mathrm{m}^{3}\right)$
$D: \quad$ Dispersion coefficient in the reactor $\left(\mathrm{m}^{2} / \mathrm{h}\right)$

$D_{e}: \quad$ Effective diffusion coefficient of the pollutant in the biolayer $\left(\mathrm{m}^{2} / \mathrm{h}\right)$

$H$ : $\quad$ Reactor height $(\mathrm{m})$

$K_{I}: \quad$ Inhibition constant $\left(\mathrm{g} / \mathrm{m}^{3}\right)$

$K_{s}$ : $\quad$ Saturation constant in the specific growth rate expression of a culture growing on the pollutant $\left(\mathrm{g} / \mathrm{m}^{3}\right)$

$N_{t}$ : Number of experimental points

$N_{w}$ : Number of neural model parameters

$R^{2}$ : Coefficient of determination

RE: Removal efficiency

$S_{G}$ : Dimensionless pollutant concentration in the gas phase $\left(C_{G} / C_{G 0}\right)$

$S_{F}$ : Dimensionless pollutant concentration in the biological phase $\left(C_{F} / C_{G 0}\right)$

$U_{g}: \quad$ Superficial gas velocity $(\mathrm{m} / \mathrm{h})$

$Y: \quad$ Yield coefficient of the culture on the pollutant (g-biomass/g-compound)

$X_{F}: \quad$ Biofilm density (g-dry cells $/ \mathrm{m}^{3}$ biofilm)

$b: \quad$ Bias term of the neural model

$f_{e}$ : Activation function of the hidden neurons

$f_{o}$ : $\quad$ Activation function of the output neurons

$f_{\mathrm{NN} 1}(z)$ : Neural network for the heterogeneous hybrid model

$f_{\mathrm{NN} 2}(z)$ : Neural network for the homogeneous hybrid model

$h$ : $\quad$ Position in the column; $h=0$ at the entrance and $h=H$ at the exit

$m_{S}$ : Maintenance coefficient (g-hexane/g-biomass/ h)

m: $\quad$ Pollutant air/biofilm distribution coefficient 
$p_{r}: \quad$ Parameter vector related to kinetics

$w_{1}(j, i)$ : Weight connecting $j$ th input and $i$ th hidden neuron

$w_{2}(j, i)$ : Weight connecting $j$ th hidden neuron and $i$ th output neuron

$x: \quad$ Dimensionless biolayer thickness $\left(\theta / \delta^{*}\right)$

$z: \quad$ Dimensionless reactor height $(h / H)$

$\mathbf{z}_{1}$ : Input vector of neural network

$\mathbf{z}_{2}$ : Signal vector of neural network from the hidden to the output layer

$\mathbf{z}_{3}$ : $\quad$ Output vector of neural network.

\section{Greek Letters}

$\alpha: \quad$ Fraction of $A$ covered by the biofilm

$\delta^{*}: \quad$ Effective biolayer thickness $(\mathrm{m})$

$\eta$ : $\quad$ Dimensionless biolayer thickness $\left(\theta / \delta^{*}\right)$

$\mu_{\max }$ : Maximum specific growth rate $\left(\mathrm{h}^{-1}\right)$ in Monod kinetics, kinetic constant in Andrews kinetics (Monod-type equation with substrate inhibition)

$v$ : $\quad$ Bed porosity position in the biolayer $(\mathrm{m}), \theta=0$ at the air/biofilm interface and $\theta=\delta^{*}$ at the biofilm/support interface

$\zeta$ : $\quad$ Dimensionless reactor height $(h / H)$.

\section{Conflict of Interests}

The authors declare that there is no conflict of interests regarding the publication of this paper.

\section{References}

[1] C. Kennes, "Biotechniques for air pollution control and bioenergy," Journal of Chemical Technology and Biotechnology, vol. 87, no. 6, pp. 723-724, 2012.

[2] D. Wu, X. Quan, Y. Zhang, and Y. Zhao, "Long-term operation of a compost-based biofilter for biological removal of $n$ butyl acetate, p-xylene and ammonia gas from an air stream," Biochemical Engineering Journal, vol. 32, no. 2, pp. 84-92, 2006.

[3] A. Zehraoui, A. A. Hassan, and G. A. Sorial, "Biological treatment of n-hexane and methanol in trickle bed air biofilters under acidic conditions," Biochemical Engineering Journal, vol. 77, pp. 129-135, 2013.

[4] S. Arriaga and S. Revah, "Removal of $n$-hexane by Fusarium solani with a gas-phase biofilter," Journal of Industrial Microbiology and Biotechnology, vol. 32, no. 11-12, pp. 548-553, 2005.

[5] S. Arriaga and S. Revah, "Mathematical modeling and simulation of hexane degradation in fungal and bacterial biofilters: effective diffusivity and partition aspects," Canadian Journal of Civil Engineering, vol. 36, no. 12, pp. 1919-1925, 2009.

[6] N. J. R. Kraakman, J. Rocha-Rios, and M. C. M. van Loosdrecht, "Review of mass transfer aspects for biological gas treatment," Applied Microbiology and Biotechnology, vol. 91, no. 4, pp. 873886, 2011.

[7] A. Vergara-Fernández, S. Hernández, and S. Revah, "Phenomenological model of fungal biofilters for the abatement of hydrophobic VOCs," Biotechnology and Bioengineering, vol. 101, no. 6, pp. 1182-1192, 2008.
[8] R. Salehahmadi, R. Halladj, and S. M. Zamir, "Unsteady-state mathematical modeling of a fungal biofilter treating hexane vapor at different operating temperatures," Industrial and Engineering Chemistry Research, vol. 51, no. 5, pp. 2388-2396, 2012.

[9] G. Spigno and D. M. de Faveri, "Modeling of a vapor-phase fungi bioreactor for the abatement of hexane: fluid dynamics and kinetic aspects," Biotechnology and Bioengineering, vol. 89, no. 3, pp. 319-328, 2005.

[10] S. Bordel, R. Muñoz, L. F. Díaz, and S. Villaverde, "Mechanistic model for evaluating the performance of suspended growth bioreactors for the off-gas treatment of VOCs," Biochemical Engineering Journal, vol. 38, no. 3, pp. 395-405, 2008.

[11] A. D. Dorado, G. Baquerizo, J. P. Maestre, X. Gamisans, D. Gabriel, and J. Lafuente, "Modeling of a bacterial and fungal biofilter applied to toluene abatement: kinetic parameters estimation and model validation," Chemical Engineering Journal, vol. 140, no. 1-3, pp. 52-61, 2008.

[12] G. D. Bellos, L. E. Kallinikos, C. E. Gounaris, and N. G. Papayannakos, "Modelling of the performance of industrial HDS reactors using a hybrid neural network approach," Chemical Engineering and Processing, vol. 44, no. 5, pp. 505-515, 2005.

[13] N. Bhutani, G. P. Rangaiah, and A. K. Ray, "First-principles, data-based, and hybrid modeling and optimization of an industrial hydrocracking unit," Industrial and Engineering Chemistry Research, vol. 45, no. 23, pp. 7807-7816, 2006.

[14] S. Tronci, R. Baratti, and A. Servida, "Monitoring pollutant emissions in a $4.8 \mathrm{MW}$ power plant through neural network," Neurocomputing, vol. 43, no. 1-4, pp. 3-15, 2002.

[15] L. Gong, Y. Xi, and C. Liu, "Embedded artificial neuval networkbased real-time half-wave dynamic resistance estimation during the A.C. resistance spot welding process," Mathematical Problems in Engineering, vol. 2013, Article ID 862076, 7 pages, 2013.

[16] I. Chairez, I. García-Peña, and A. Cabrera, "Dynamic numerical reconstruction of a fungal biofiltration system using differential neural network," Journal of Process Control, vol. 19, no. 7, pp. 1103-1110, 2009.

[17] A. Elías, G. Ibarra-Berastegi, R. Arias, and A. Barona, "Neural networks as a tool for control and management of a biological reactor for treating hydrogen sulphide," Bioprocess and Biosystems Engineering, vol. 29, no. 2, pp. 129-136, 2006.

[18] E. R. Rene, M. C. Veiga, and C. Kennes, "Experimental and neural model analysis of styrene removal from polluted air in a biofilter," Journal of Chemical Technology and Biotechnology, vol. 84, no. 7, pp. 941-948, 2009.

[19] E. R. Rene, J. H. Kim, and H. S. Park, "Immobilized cell biofilter: results of performance and neural modeling strategies for $\mathrm{NH}_{3}$ vapor removal from waste gases," Aerosol and Air Quality Research, vol. 9, no. 3, pp. 379-384, 2009.

[20] E. R. Rene, M. Estefanía López, M. C. Veiga, and C. Kennes, "Neural network models for biological waste-gas treatment systems," New Biotechnology, vol. 29, no. 1, pp. 56-73, 2011.

[21] M. Zamir, R. Halladj, M. Saber, M. Ferdowsi, and B. Nasernejad, "Biofiltration of hexane vapor: experimental and neural model analysis," Clean-Soil, Air, Water, vol. 39, no. 9, pp. 813-819, 2011.

[22] G. Porru, C. Aragonese, R. Baratti, and A. Servida, "Monitoring of a $\mathrm{CO}$ oxidation reactor through a grey model-based EKF observer," Chemical Engineering Science, vol. 55, no. 2, pp. 331$338,2000$.

[23] A. A. Safavi, A. Nooraii, and J. A. Romagnoli, "A hybrid model formulation for a distillation column and the on-line 
optimisation study," Journal of Process Control, vol. 9, no. 2, pp. 125-134, 1999.

[24] S. Tronci, M. Medde, and R. Baratti, "An hybrid model for the hydrotreatment of gasoil, ICheaP-9," Chemical Engineering Transactions, vol. 17, pp. 1233-1238, 2009.

[25] B. S. Kumar and C. Venkateswarlu, "Estimating biofilm reaction kinetics using hybrid mechanistic-neural network rate function model," Bioresource Technology, vol. 103, no. 1, pp. 300-308, 2012.

[26] A. Saraceno, S. Curcio, V. Calabrò, and G. Iorio, "A hybrid neural approach to model batch fermentation of 'ricotta cheese whey' to ethanol," Computers and Chemical Engineering, vol. 34, no. 10, pp. 1590-1596, 2010.

[27] A. Saraceno, M. Aversa, and S. Curcio, "Advanced modeling of food convective drying: a comparison between artificial neural networks and hybrid approaches," Food and Bioprocess Technology, vol. 5, no. 5, pp. 1694-1705, 2012.

[28] G. Spigno, C. Pagella, M. D. Fumi, R. Molteni, and D. M. de Faveri, "VOCs removal from waste gases: gas-phase bioreactor for the abatement of hexane by Aspergillus niger," Chemical Engineering Science, vol. 58, no. 3-6, pp. 739-746, 2003.

[29] C. Principe, N. R. Euliano, and W. C. Lefebvre, Neural and Adaptive Systems: Fundamentals Through Simulation, Wiley, New York, NY, USA, 1999.

[30] C. Kennes and M. C. Veiga, Bioreactors for Waste Gas Treatment, Kluwer Academic, Dordrecht, The Netherlands, 2001.

[31] M. B. Neumann and W. Gujer, "Underestimation of uncertainty in statistical regression of environmental models: influence of model structure uncertainty," Environmental Science and Technology, vol. 42, no. 11, pp. 4037-4043, 2008.

[32] D. C. Montgomery, Design and Analysis of Experiments, John Wiley \& Sons, Hoboken, NJ, USA, 7th edition, 2008.

[33] R. B. D'Agostino and M. A. Stephens, Goodness-of-Fit Techniques, Marcel Dekker, New York, NY, USA, 1986.

[34] M. Grosso, O. Galan, R. Baratti, and J. A. Romagnoli, "On the prediction and shaping of the PSD in crystallization operations," Computer Aided Chemical Engineering, vol. 28, pp. 805-810, 2010. 


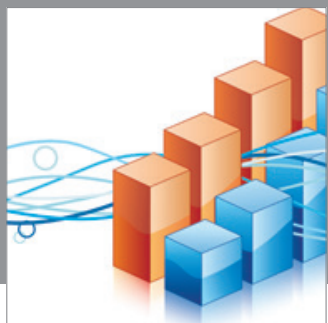

Advances in

Operations Research

mansans

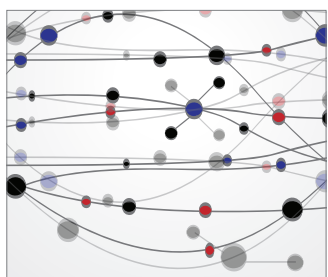

The Scientific World Journal
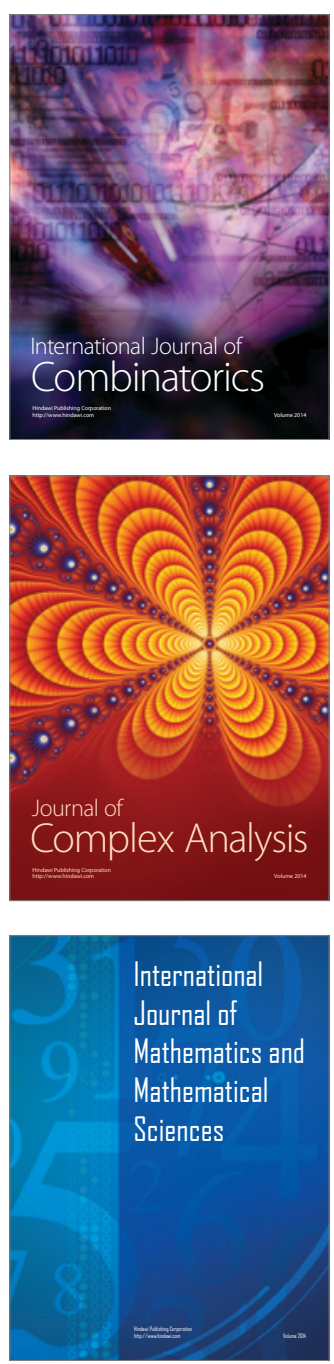
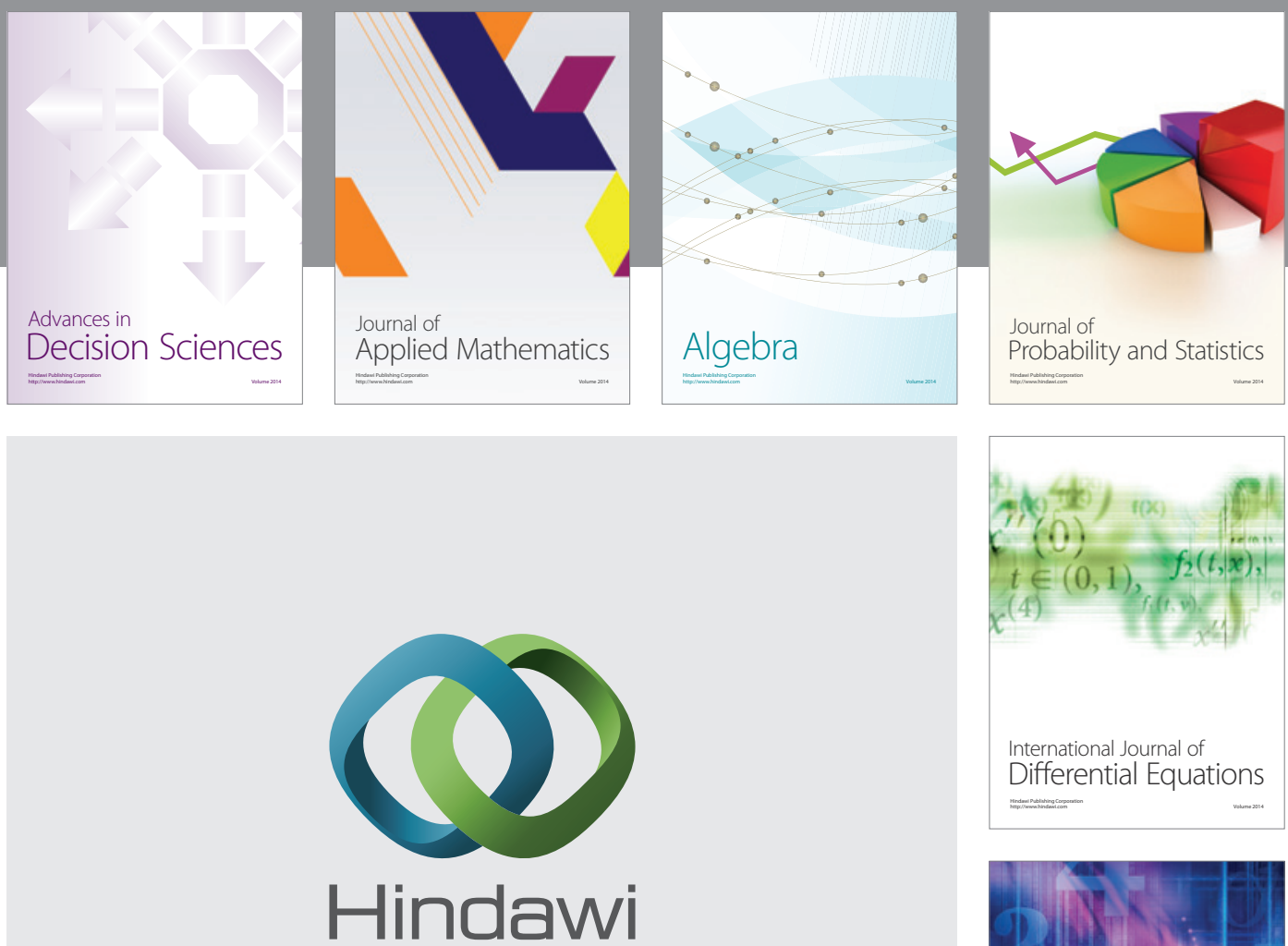

Submit your manuscripts at http://www.hindawi.com
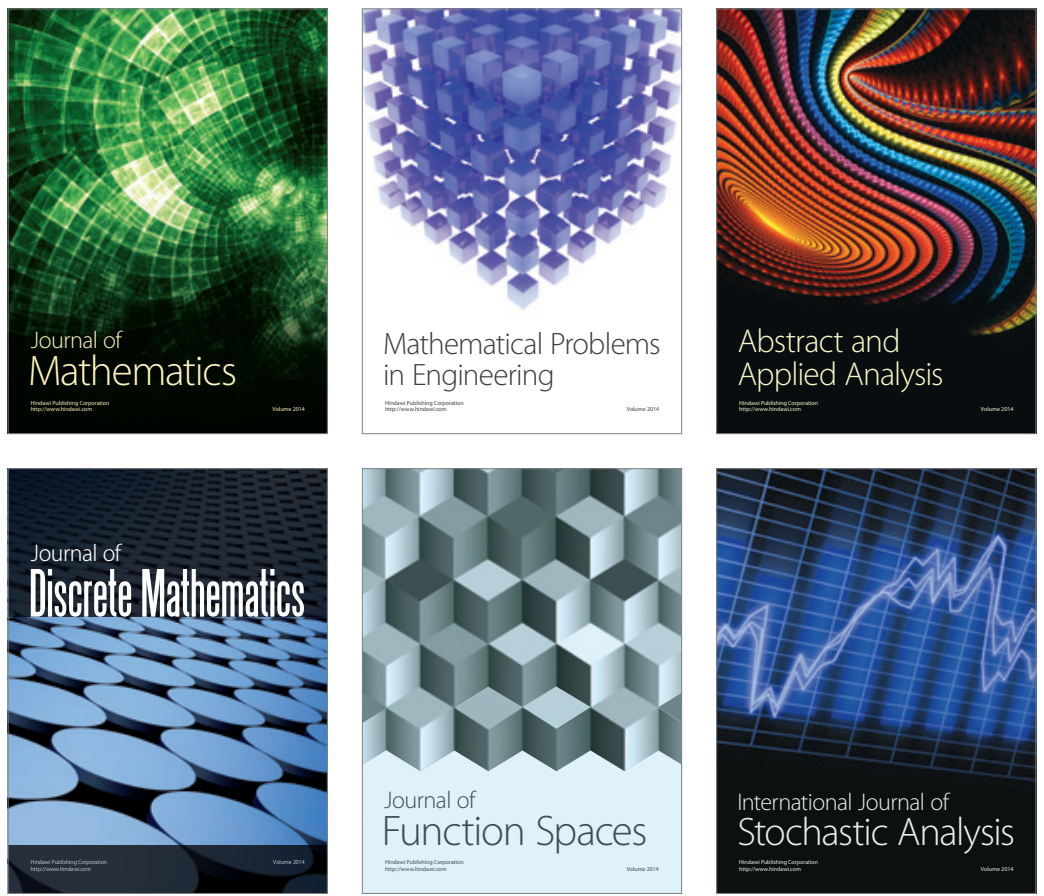

Journal of

Function Spaces

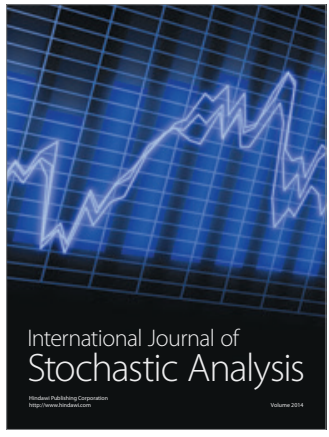

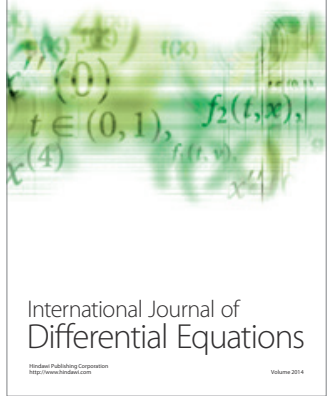
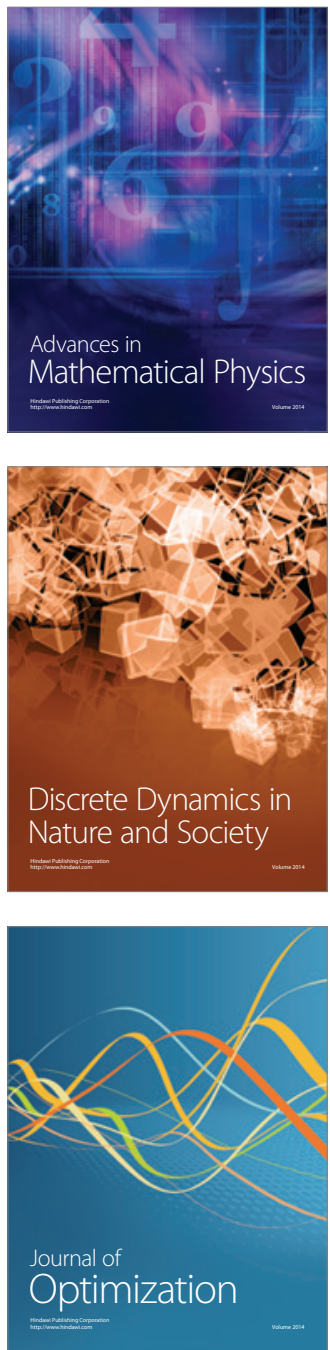